\title{
Exchange-only optimized-effective-potential calculations using Slater-type basis functions: Atoms and diatomic molecules
}

\author{
J. J. Fernández ${ }^{*}$ and J. E. Alvarellos \\ Departamento de Física Fundamental, UNED, Apartado 60.141, E-28080 Madrid, Spain \\ P. García-González \\ Departamento de Física Teórica de la Materia Condensada, Universidad Autónoma de Madrid, Cantoblanco, E-28049 Madrid, Spain \\ M. Filatov \\ Department of Theoretical Chemistry, Zernike Institute for Advanced Materials, University of Groningen, \\ Nijenborgh 4, 9747 AG Groningen, Netherlands \\ (Received 22 November 2011; published 17 January 2012)
}

\begin{abstract}
The exchange-only optimized-effective-potential method is implemented with the use of Slater-type basis functions, seeking an alternative to the standard methods of solution with some computational advantages. This procedure has been tested in a small group of closed-shell atoms and diatomic molecules, for which numerical solutions are available. The results obtained with this implementation have been compared to the exact numerical solutions and to the results obtained when the optimized effective equations are solved using the Gaussian-type basis sets. This Slater-type basis approach leads to a more compact expansion space for representing the potential of the optimized-effective-potential method and to considerable computational savings when compared to both the numerical solution and the more traditional one in terms of the Gaussian basis sets.
\end{abstract}

DOI: 10.1103/PhysRevA.85.012512

PACS number(s): 31.15.xr, 31.15.E-

\section{INTRODUCTION}

In practical implementations of the Kohn-Sham density functional theory $[1,2]$ the exchange-correlation (XC) energy is usually described by a suitable explicit functional of the electron density and the parameters characterizing the density inhomogeneity. A very promising step to improve the accuracy of density functional calculations relies on the use of orbitaldependent density functionals, in which the XC energy is expressed as an explicit functional of Kohn-Sham (KS) orbitals [3]. A rigorous approach to implement the orbital-dependent functionals within the $\mathrm{KS}$ formalism is the optimizedeffective-potential (OEP) method [3-6], where the XC potential is described by a local multiplicative term and the total energy functional is orbital dependent. By virtue of the Hohenberg-Kohn theorems [1], the OEP solution is equivalent to the minimization of the total electronic energy with respect to the density [7]. In the case of the exchange-only-i.e., the Hartree-Fock (HF) — energy functional, the corresponding OEP (xOEP) method was first formulated by Sharp and Horton [4] and numerically solved in real space for atoms by Talman and Shadwick [5]. Applications of the real-space xOEP formalism to atoms, molecules, and solids, thanks to the use of pseudopotentials, have been reported in the literature [8-14]. A number of approximations to the xOEP method, such as the Krieger-Li-Iafrate method [15,16], the local HF method [17], and the effective-local-potential [18] method, have been developed.

The real-space resolution of the xOEP method has only been efficiently applied to highly symmetric systems, such as spherically symmetric atoms and diatomic molecules $[5,9]$.

*jjfernandez@ fisfun.uned.es
Application of the xOEP formalism to polyatomic molecules requires its formulation in terms of basis sets suitable for molecular calculations. Currently, there exist several formulations of the XOEP method in terms of basis sets of local Gaussian-type-orbital (GTO) functions [19-23]. The most popular implementation of the XOEP formalism employs two different basis sets, one for the expansion of the KS orbitals and another one for representing the local multiplicative potential $[22,24,25]$. Within this approach, special care must be taken when selecting the auxiliary basis set for the potential, thus leading to the concept of a balanced basis set firmly connected to the orbital basis set [25]. Alternatively, a set of the products of the occupied and virtual KS orbitals can be employed for the solution of the XOEP equations for the local potential $[19,20,26]$.

The computational complexity of the xOEP method in a basis-set representation depends critically on the size of the orbital-expansion basis set. For obtaining faithful solutions of the xOEP equations, the orbital basis set should support the linear dependence in the space of the occupied-virtual orbital products $[19,23,27]$. With the use of GTO basis functions, this requirement leads to very large orbital basis sets with hundreds of basis functions even for small molecules. On the other hand, the use of Slater-type-orbital (STO) basis functions gives considerably more compact orbital basis sets, which can be beneficial for the application of the xOEP formalism, and a very efficient implementation of quantum chemical formalisms with Slater-type basis functions has been achieved in the SMILES suite of programs [28].

It is the primary purpose of the present work to implement the XOEP method within the SMILES package and to analyze the advantages that can be obtained from the use of the STO basis sets, testing it for a small number of atoms and diatomic molecules, for which both numerical solutions $[8,9]$ 
and STOs are available [28]. In this work we employ the xOEP algorithm outlined in Refs. [20,21] and the xOEP equations, formulated in terms of the STOs. The solution of the xOEP equation is carried out through the truncated singular-valuedecomposition (TSVD) technique. It is demonstrated that the use of the Slater-type basis sets leads to considerable computational savings in every step of the self-consistent procedure, without deteriorating the accuracy of the calculated xOEP total and orbital energies.

\section{THEORY}

In this section the main features of the OEP method are outlined. In the OEP method one seeks a local multiplicative potential $V_{\sigma}(\mathbf{r})$ such that its eigenfunctions (atomic units are used in this paper)

$$
\left(-\frac{1}{2} \nabla^{2}+V_{\sigma}(\mathbf{r})\right) \phi_{p \sigma}=\epsilon_{p \sigma} \phi_{p \sigma}
$$

minimize the total energy functional given by

$$
\begin{aligned}
& E^{\mathrm{OEP}}\left[\left\{\phi_{i \sigma}\right\}\right] \\
& =\sum_{\sigma} \sum_{i} \int \phi_{i \sigma}^{*}(\mathbf{r})\left(-\frac{1}{2} \nabla^{2}\right) \phi_{i \sigma}(\mathbf{r}) d \mathbf{r}+\int \rho(\mathbf{r}) V_{\mathrm{ext}}(\mathbf{r}) d \mathbf{r} \\
& \quad+\frac{1}{2} \int \rho(\mathbf{r}) \int \rho\left(\mathbf{r}^{\prime}\right) \frac{1}{\left|\mathbf{r}-\mathbf{r}^{\prime}\right|} d \mathbf{r} d \mathbf{r}^{\prime}+E_{\mathrm{XC}}\left[\left\{\phi_{i \sigma}\right\}\right]
\end{aligned}
$$

where $\sum_{i}$ runs over occupied orbitals and $\sum_{\sigma}$ over the spin, where $\rho(\mathbf{r})=\sum_{i} \sum_{\sigma}\left|\phi_{i \sigma}\right|^{2}$ is the electron density and $E_{\mathrm{XC}}\left[\left\{\phi_{i \sigma}\right\}\right]$ is the orbital-dependent exchange-correlation energy. Here and below we use indices $i, j, \ldots$ for occupied orbitals, $a, b, \ldots$ for unoccupied orbitals, and $p, q, \ldots$ for general (i.e., occupied or unoccupied) orbitals. In the case of the exchange-only formalism, xOEP, the XC energy $E_{\mathrm{XC}}\left[\left\{\phi_{i \sigma}\right\}\right]$ is replaced with the HF exchange energy,

$$
E_{x}\left[\left\{\phi_{i \sigma}\right\}\right]=-\frac{1}{2} \sum_{i, j} \sum_{\sigma} \int \frac{\phi_{i \sigma}^{*}(\mathbf{r}) \phi_{j \sigma}(\mathbf{r}) \phi_{j \sigma}^{*}\left(\mathbf{r}^{\prime}\right) \phi_{i \sigma}\left(\mathbf{r}^{\prime}\right)}{|\mathbf{r}-\mathbf{r}|^{\prime}} .
$$

The local multiplicative potential

$$
V_{\sigma}(\mathbf{r})=V_{\text {ext }}(\mathbf{r})+\int \frac{\rho\left(\mathbf{r}^{\prime}\right)}{\left|\mathbf{r}-\mathbf{r}^{\prime}\right|} d \mathbf{r}^{\prime}+V_{\sigma}^{x}(\mathbf{r})
$$

is split into the external potential $V_{\text {ext }}(\mathbf{r})$ (i.e. the potential due to the nuclei), the Coulomb potential of the electron cloud, and the local exchange potential $V_{\sigma}^{x}(\mathbf{r})$.

The $x O E P$ equations in a basis-set representation are obtained from the minimization of the total energy presented in Eq. (2) with respect to the local potential $V_{\sigma}(\mathbf{r})[25,29]$, this minimization being equivalent to the minimization with respect to the density by virtue of the Sham-Schlüter condition [7] and the Hohenberg-Kohn theorems [1].

An expansion of the exchange part of the local potential is assumed in terms of an appropriate set of functions $[20,22,24,25,29,30]$,

$$
V_{\sigma}^{x}(\mathbf{r})=\sum_{\mu} \tilde{w}_{\mu \sigma} f_{\mu \sigma}(\mathbf{r})
$$

where $\sigma$ denotes the spin, $\mu$ denotes all needed values of the basis index, and $\tilde{w}_{\mu \sigma}$ are the expansion coefficients of the exchange potential in this basis. Following the literature [20], the expansion functions are conveniently defined as

$$
f_{\mu \sigma}(\mathbf{r})=\int \frac{g_{\mu \sigma}\left(\mathbf{r}^{\prime}\right)}{\left|\mathbf{r}-\mathbf{r}^{\prime}\right|} d \mathbf{r}^{\prime}
$$

where $g_{\mu \sigma}(\mathbf{r})$ are square-integrable functions. Note that this definition implies that the expansion functions $f_{\mu \sigma}(\mathbf{r})$ are not necessarily square integrable; this is not a problem as the local potential does not satisfy this condition.

The requirement that the total energy be stationary under the variations of the local potential, i.e., $\delta E^{\mathrm{xOEP}} / \delta V_{\sigma}(\mathbf{r})=0$, is then equivalent to finding a minimum of the total $\mathrm{xOEP}$ energy with respect to the set of the expansion coefficients of the local potential, $\left\{\tilde{w}_{\mu \sigma}\right\}$. If we work with a real orbital basis and if we introduce a scalar product of two functions $h$ and $l$ of our expansion space as

$$
(h \mid l)=\iint h(\mathbf{r}) \frac{1}{\left|\mathbf{r}-\mathbf{r}^{\prime}\right|} l\left(\mathbf{r}^{\prime}\right) d \mathbf{r} d \mathbf{r}^{\prime},
$$

the minimization of the $\mathrm{xOEP}$ energy-i.e., Eq. (2) with $E_{\mathrm{XC}}$ defined as the Fock exchange energy given in Eq. (3)-leads to the equation [21]

$$
\begin{aligned}
\frac{\partial E^{\mathrm{xOEP}}}{\partial \tilde{w}_{\mu \sigma}}= & 2 \sum_{i a} \frac{\left(g_{\mu \sigma} \mid \phi_{a \sigma} \phi_{i \sigma}\right)}{\varepsilon_{a \sigma}-\varepsilon_{i \sigma}} \iint d \mathbf{r} d \mathbf{r}^{\prime} \phi_{i \sigma}\left(\mathbf{r}^{\prime}\right) \\
& \times\left[V_{\sigma}^{x}\left(\mathbf{r}^{\prime}\right)-V_{\sigma}^{x, \mathrm{nl}}\left(\mathbf{r}, \mathbf{r}^{\prime}\right)\right] \phi_{a \sigma}\left(\mathbf{r}^{\prime}\right)=0 .
\end{aligned}
$$

Here the nonlocal potential, $V_{\sigma}^{x, \mathrm{nl}}\left(\mathbf{r}, \mathbf{r}^{\prime}\right)$, is defined as

$$
V_{\sigma}^{x, \mathrm{nl}}\left(\mathbf{r}, \mathbf{r}^{\prime}\right) \phi_{j \sigma}\left(\mathbf{r}^{\prime}\right)=\frac{\delta E_{x}\left[\left\{\phi_{i \sigma}(\mathbf{r})\right\}\right]}{\delta \phi_{j \sigma}\left(\mathbf{r}^{\prime}\right)}
$$

where $\left\{\phi_{q \sigma}\right\}$ are the solutions of Eq. (1).

Using the matrix

$$
M_{\mu, j b}^{\sigma}=\frac{\left(g_{\mu \sigma} \mid \phi_{b \sigma} \phi_{j \sigma}\right)}{\sqrt{\varepsilon_{b \sigma}-\varepsilon_{j \sigma}}}
$$

in Eq. (8) we get a matrix equation equivalent to the minimization of the xOEP energy,

$$
\nabla_{\tilde{\mathbf{w}}} E^{\mathrm{xOEP}}=2 \mathbf{M M}^{\dagger} \tilde{\mathbf{w}}-2 \mathbf{M} \mathbf{w}^{\mathrm{nl}}=0,
$$

where $\tilde{\mathbf{w}}$ is the vector of the expansion coefficients for the local exchange potential and $\mathbf{w}^{\mathrm{nl}}$ is the projection in the chosen basis set of the nonlocal HF potential, $\hat{V}_{\sigma}^{x, \mathrm{nl}}\left(\mathbf{r}, \mathbf{r}^{\prime}\right)$.

In this work our expansion basis set is a scaled form of the occupied-virtual products, specifically [20,21,23,26]

$$
g_{\mu \sigma}(\mathbf{r})=\frac{\phi_{a \sigma}(\mathbf{r}) \phi_{i \sigma}(\mathbf{r})}{\sqrt{\varepsilon_{a \sigma}-\varepsilon_{i \sigma}}} .
$$

So, in Eq. (5) the summation over $\mu$ runs over products of an occupied orbital $i$ and an unoccupied one $a$. Thus, the elements of the vector $\mathbf{w}^{\mathrm{nl}}$ are

$$
w_{a i \sigma}^{\mathrm{nl}}=\int d \mathbf{r} d \mathbf{r}^{\prime} \frac{\phi_{a \sigma}(\mathbf{r}) \phi_{i \sigma}\left(\mathbf{r}^{\prime}\right)}{\sqrt{\varepsilon_{a \sigma}-\varepsilon_{i \sigma}}} V_{\sigma}^{x, \mathrm{nl}}\left(\mathbf{r}, \mathbf{r}^{\prime}\right),
$$

and the matrix elements (10) reduce to

$$
M_{i a, j b}^{\sigma}=\frac{\left(\phi_{a \sigma} \phi_{i \sigma} \mid \phi_{b \sigma} \phi_{j \sigma}\right)}{\sqrt{\varepsilon_{a \sigma}-\varepsilon_{i \sigma}} \sqrt{\varepsilon_{b \sigma}-\varepsilon_{j \sigma}}} .
$$

Note that with the basis set of occupied-virtual products it is not possible to get any term having a $1 / r$ asymptotic decay. This is corrected by the addition to our basis set $\left\{g_{\mu \sigma}(\mathbf{r})\right\}$ of 
TABLE I. Total energies (in hartrees) for the Be atom using the HF and the xOEP methods with the STO and the GTO basis sets. In the first column we indicate the value $\varepsilon$ used as a threshold in the TSVD decomposition of the $\mathbf{M M}^{\dagger}$ matrix in the xOEP method. For the STO and GTO xOEP results we have included, within parentheses, the total number of occupied-virtual products (first number in parentheses) and the number of them that are used (second number in parentheses) in each calculation.

\begin{tabular}{lccrr}
\hline \hline$\varepsilon$ & STO basis & $E_{\text {xOEP }}^{\text {STO }}$ & GTO basis & $E_{\text {xOEP }}^{\text {GTO }}$ \\
\hline $10^{-3}$ & VB1 & $-14.57238(12 / 3)$ & cc-pVTZ & $-14.57233(26 / 10)$ \\
& VB2 & $-14.57240(17 / 4)$ & cc-pVQZ & $-14.57234(38 / 17)$ \\
$10^{-4}$ & VB3 & $-14.57240(22 / 5)$ & cc-pV5Z & $-14.57227(52 / 20)$ \\
& VB1 & $-14.57242(12 / 4)$ & cc-pVTZ & $-14.57233(26 / 14)$ \\
& VB2 & $-14.57246(17 / 5)$ & cc-pVQZ & $-14.57256(38 / 20)$ \\
$10^{-5}$ & VB3 & $-14.57245(22 / 7)$ & cc-pV5Z & $-14.57255(52 / 26)$ \\
& VB1 & $-14.57244(12 / 6)$ & cc-pVTZ & $-14.57241(26 / 16)$ \\
& VB2 & $-14.57256(17 / 8)$ & cc-pVQZ & $-14.57256(38 / 21)$ \\
& VB3 & $-14.57254(22 / 13)$ & cc-pV5Z & $-14.57255(52 / 30)$ \\
HF & STO basis & $E_{\mathrm{HF}}^{\text {STO }}$ & GTO basis & cc-pVTZ \\
& VB1 & -14.57297 & cc-pVQZ & -14.57287 \\
& VB2 & -14.57298 & cc-pV5Z & -14.57296 \\
\hline \hline
\end{tabular}

the Fermi-Amaldi function $s(\mathbf{r})=\rho(\mathbf{r}) / N$, where $N$ is the number of electrons of the system. That procedure reproduces the Fermi-Amaldi potential for long distances and makes the xOEP highest occupied molecular orbital eigenenergies very close, but not equal, to those found using the HF method. This prescription [24] is very different from the one adopted when the exchange potential is expanded in an auxiliary basis set [17].

\section{COMPUTATIONAL DETAILS}

As the products of the occupied and virtual states are linearly dependent, the matrix $\mathbf{M M}^{\dagger}$ appearing in Eq. (11) is singular and the equation cannot be solved by inversion [25]. Following the argument given in Refs. [31] and [27], only the linearly independent functions $f_{\mu \sigma}(\mathbf{r})$ [or $\left.g_{\mu \sigma}(\mathbf{r})\right]$ can be used in the expansion of the xOEP; i.e., for a faithful solution of the $\mathrm{xOEP}$ equation (11), linearly independent orbital products must be employed. We then apply the TSVD technique to separate the linearly dependent occupied-virtual products and the independent ones, seeking the linearly independent set of products by diagonalization of the $\mathbf{M} \mathbf{M}^{\dagger}$ matrix. In order to fulfill this condition, a threshold is chosen to discriminate the elements of the $\mathbf{M M}^{\dagger}$ matrix that correspond to the linearly independent functions. ${ }^{1}$ In this way, the mapping between the density and density matrix becomes nonunique and a solution with the energy $E_{\mathrm{xOEP}}>E_{\mathrm{HF}}$ is obtained [27,32].

In our case, the TSVD method requires the diagonalization of the matrix $\mathbf{M M}^{\dagger}$, in general a very large one. But as the STOs represent the unoccupied orbitals in a much more efficient way than the GTOs, when Slater-type-orbital basis sets are used the size of the matrix $\mathbf{M} \mathbf{M}^{\dagger}$, and consequently the expansion space

\footnotetext{
${ }^{1}$ If all the orbital products were linearly independent (thus making the matrix $\mathbf{M} \mathbf{M}^{\dagger}$ perfectly invertible), then Eq. (11) would have had a unique solution that would correspond to the lowest variational energy obtainable with the functional given in Eq. (3), that is, the HF energy $E_{\mathrm{HF}}$.
}

for the local potential in Eq. (5), is much smaller than when using GTOs. This is the main point of this paper: when STO basis sets are used, the computational effort (in memory size and in speed of the calculations) for each self-consistent cycle is much less, whereas the quality of the results is preserved.

The algorithm outlined in the previous section was implemented in the SMILES suite of programs [28], which employs the STO basis sets in quantum-chemical calculations. We compare the results of the XOEP calculations obtained with the Slater-type basis functions (xOEP-STO) to both numerical exact solutions and $\mathrm{xOEP}$ results obtained with the use of the Gaussian-type basis functions (xOEP-GTO). The latter results were obtained with the use of the MOLPRO2008.1 code [33], where the XOEP formalism employing Gaussian-type basis sets was recently implemented by some of us [20,21]. In order to use comparable basis sets, the correlation-consistent basis sets of Dunning (cc-pVTZ, cc-pVQZ, and cc-pV5Z) were used in the xOEP-GTO calculations, and the STOs of similar quality (VB1 for cc-pVTZ, VB2 for cc-pVQZ, and VB3 for cc-pV5Z) [28] were selected for the XOEP-STO calculations. These STO and GTO basis sets yield the total HF energies in close agreement (see Tables I, II, and III below). All the basis sets we have used (xOEP-STO and xOEP-GTO calculations) were employed in their uncontracted form.

Due to the few exact numerical xOEP solutions found in the literature, and to the small number of available STOs for atomic and molecular computations, the calculations we present here were performed for the $\mathrm{Be}$ and $\mathrm{Ne}$ atoms and for the $\mathrm{LiH}, \mathrm{BH}$, $\mathrm{Li}_{2}$, and $\mathrm{CO}$ molecules. The numerical solutions were given by Makmal et al. [9] and we have used the same internuclear distances (in a.u.): 3.015 for $\mathrm{LiH}, 2.336$ for $\mathrm{BH}, 5.051$ for $\mathrm{Li}_{2}$, and 2.132 for $\mathrm{CO}$. This distance for the $\mathrm{CO}$ molecule has also been used by Heßelmann et al. [22] in the xOEP-GTO solution, and we also compare our results with theirs in Sec. IV.

\section{RESULTS}

The dependence of the xOEP-STO and the xOEP-GTO total energies on the size of the basis set and on the TSVD 
TABLE II. Total energies (in hartrees) for the LiH molecule using the HF and the xOEP methods with the STO and the GTO basis sets. In the first column we indicate the value $\varepsilon$ used as a threshold in the TSVD decomposition of the $\mathbf{M M}^{\dagger}$ matrix in the xOEP method. For the xOEP-STO and xOEP-GTO results we have included, within parentheses, the total number of occupied-virtual products (first number within parentheses) and the number of them that are used (second number within parentheses) in each calculation.

\begin{tabular}{lcccc}
\hline \hline$\varepsilon$ & STO basis & $E_{\text {xOEP }}^{\text {STO }}$ & GTO basis & $E_{\text {xOEP }}^{\text {GTO }}$ \\
\hline $10^{-3}$ & VB1 & $-7.98651(14 / 3)$ & cc-pVTZ & $-7.98660(58 / 24)$ \\
& VB2 & $-7.98660(18 / 4)$ & cc-pVQZ & $-7.98661(88 / 32)$ \\
$10^{-4}$ & VB3 & $-7.98669(26 / 6)$ & cc-pV5Z & $-7.98671(134 / 42)$ \\
& VB1 & $-7.98658(14 / 6)$ & cc-pVTZ & $-7.98661(58 / 29)$ \\
& VB2 & $-7.98693(18 / 7)$ & cc-pVQZ & $-7.98698(88 / 44)$ \\
$10^{-5}$ & VB3 & $-7.98693(26 / 9)$ & cc-pV5Z & $-7.98696(134 / 63)$ \\
& VB1 & $-7.98681(14 / 8)$ & cc-pVTZ & $-7.98678(58 / 36)$ \\
& VB2 & $-7.98699(18 / 9)$ & cc-pVQZ & $-7.98701(88 / 52)$ \\
& VB3 & $-7.98689(26 / 13)$ & cc-pV5Z & $-7.98700(134 / 74)$ \\
HF & STO basis & $E_{\mathrm{HF}}^{\text {STO }}$ & GTO basis & cc-pVTZ \\
& VB1 & -7.98479 & cc-pVQZ & -7.98695 \\
& VB2 & -7.98650 & cc-pV5Z & -7.98722 \\
\hline \hline
\end{tabular}

cutoff criterion $\varepsilon$ for neglecting (near) zero eigenvalues of the matrix $\mathbf{M M}^{\dagger}$ has been investigated. The results are collected in Tables I, II, and III. In the calculations, the TSVD cutoff criterion $\varepsilon$ was varied in the range $10^{-2}-10^{-6}$. Note that when $\varepsilon=10^{-2}$ is employed, the expansion space for the potential is very small because only a few eigenvalues of the matrix $\mathbf{M M}^{\dagger}$ are greater than $\varepsilon$, yielding energies noticeably above the numeric xOEP values. For that reason, we have not reported those energies in the tables. Reducing the TSVD cutoff $\varepsilon$ makes the expansion space bigger and, as a consequence, the total $\mathrm{xOEP}-\mathrm{STO}$ and $\mathrm{xOEP}-\mathrm{GTO}$ energies decrease, approaching the accurate numeric xOEP values. For $\varepsilon$ in the range $10^{-3}-10^{-5}$, the total $\mathrm{xOEP}$ energies remain constant to within a fraction of mHa. When the xOEP-GTO method is used with a very tight cutoff criterion $\left(\varepsilon \leqslant 10^{-6}\right)$, the iterative solution of the xOEP equation (11) becomes unstable and the xOEP total energy collapses toward the HF energy. As a matter of fact, the procedure breaks down as the matrix $\mathbf{M M}^{\dagger}$ becomes noninvertible. On the other hand, the xOEP-STO implementation shows somewhat greater stability and begins to break down at smaller values of $\varepsilon$, i.e., when $\varepsilon \leqslant 10^{-7}$. This can be attributed to the fact that, with the use of STO functions, the expansion set of the potential is much smaller than with the more traditional Gaussian-basis sets (see below).

The number of the eigenfunctions of the matrix $\mathbf{M} \mathbf{M}^{\dagger}$ used for the expansion of the potential is also given in Tables I, II, and III, as well as the total dimension of the matrix. It is seen that the dimension of $\mathbf{M M}^{\dagger}$ is considerably smaller in the XOEP-STO method, and the dimension of the potential-expansion space does not grow as fast as in the case of the xOEP-GTO method. So, the xOEP-STO implementation

TABLE III. Total energies (in hartrees) for the CO molecule using the HF and the xOEP methods using the STO and the GTO basis sets. In the first column we indicate the value $\varepsilon$ used as a threshold in the TSVD decomposition of the MM $\mathbf{M}^{\dagger}$ matrix in the xOEP method. For the xOEP-STO and the xOEP-GTO results we have included, within parentheses, the total number of occupied-virtual products (first number within parentheses) and the number of them that are used (second number within parentheses) in each calculation.

\begin{tabular}{lcccc}
\hline \hline$\varepsilon$ & STO basis & $E_{\text {xOEP }}^{\text {STO }}$ & GTO basis & $E_{\text {xOEP }}$ \\
\hline $10^{-3}$ & VB1 & $-112.77681(93 / 10)$ & cc-pVTZ & $-112.77573(438 / 94)$ \\
& VB2 & $-112.78251(111 / 13)$ & cc-pVQZ & $-112.78246(686 / 104)$ \\
& VB3 & $-112.78433(184 / 21)$ & cc-pV5Z & $-112.78428(1046 / 116)$ \\
$10^{-4}$ & VB1 & $-112.77999(93 / 16)$ & cc-pVTZ & $-112.77657(438 / 128)$ \\
& VB2 & $-112.78469(111 / 20)$ & cc-pVQZ & $-112.78392(686 / 176)$ \\
& VB3 & $-112.78521(184 / 25)$ & cc-pV5Z & $-112.78519(1046 / 238)$ \\
$10^{-5}$ & VB1 & $-112.77780(93 / 24)$ & cc-pVTZ & $-112.77704(438 / 160)$ \\
& VB2 & $-112.78524(111 / 27)$ & cc-pVQZ & $-112.78464(686 / 226)$ \\
& VB3 & $-112.78526(184 / 32)$ & cc-pV5Z & $-112.78551(1046 / 312)$ \\
& STO basis & $E_{\mathrm{HF}}^{\text {STO }}$ & GTO basis & $E_{\mathrm{HF}}^{\mathrm{GTO}}$ \\
$\mathrm{HF}$ & VB1 & -112.78199 & cc-pVTZ & -112.78014 \\
& VB2 & -112.78959 & cc-pVQZ & -112.78891 \\
& VB3 & -112.79056 & cc-pV5Z & -112.79064 \\
\hline
\end{tabular}


TABLE IV. Total xOEP energies (in hartrees) for several atomic and diatomic systems calculated using a TSVD decomposition of the $\mathbf{M M}^{\dagger}$ matrix in the xOEP method (the threshold $\varepsilon$ used to discriminate the eigenvalues was $10^{-5}$ ).

\begin{tabular}{lrrrr}
\hline \hline & Numeric & STO (VB3) & GTO (cc-pV5Z) & GTO (Hess) \\
\hline $\mathrm{Be}$ & $-14.57254^{\mathrm{b}}$ & -14.57254 & -14.57255 & -14.57243 \\
$\mathrm{Ne}$ & $-128.54553^{\mathrm{c}}$ & -128.54540 & -128.54548 & -128.54538 \\
$\mathrm{LiH}$ & $-7.98691^{\mathrm{c}}$ & -7.98689 & -7.98700 & \\
$\mathrm{Li}_{2}$ & $-14.87076^{\mathrm{c}}$ & -14.87044 & -14.87090 & \\
$\mathrm{BH}$ & $-25.12963^{\mathrm{c}}$ & -25.12941 & -25.13013 & \\
$\mathrm{CO}$ & $-112.785(3)^{\mathrm{c}}$ & -112.78526 & -112.78551 & -112.78491 \\
\hline \hline
\end{tabular}

${ }^{\text {aReference [22]. }}$

${ }^{\mathrm{b}}$ Reference [8].

${ }^{\mathrm{c}}$ Reference [9].

gives a noticeable memory savings and a greater stability with respect to the cutoff criterion of the TSVD procedure.

For all the systems in Tables I, II, and III, the differences between the xOEP-STO and the xOEP-GTO total energies are typically smaller than $1 \mathrm{mHa}$. When large basis sets are used, the total $\mathrm{xOEP}-\mathrm{STO}$ and $\mathrm{xOEP}-\mathrm{GTO}$ energies approach the exact numeric values with an accuracy better than $100 \mu \mathrm{Ha}$. It is important to stress here that the xOEP and HF total energies converge in a somewhat different way with respect to the basis-set size (remember that the basis sets we use in this paper were neither developed nor optimized for the xOEP calculations) so the differences $E_{\mathrm{xOEP}}-E_{\mathrm{HF}}$ oscillate.

Table IV summarizes the results of calculations for $\mathrm{Be}$ and $\mathrm{Ne}$ atoms and a number of diatomic molecules studied by Makmal et al. [9] using the real-space xOEP method. For the sake of comparison, the xOEP energies obtained by Heßelmann et al. [22] with the use of balanced auxiliary basis sets for the potential are also shown when available. Note that the $\mathrm{xOEP}-\mathrm{STO}$ energies are typically in a somewhat better agreement with the numerical values than the XOEP-GTO energies obtained with a similar basis set (the STO basis sets give results about $0.1 \mathrm{mHa}$ below the energies obtained by Heßelmann et al.).

Table $\mathrm{V}$ collects the energies of the occupied orbitals obtained with the use of the xOEP-STO and XOEP-GTO methods for the $\mathrm{Be}$ atoms and the $\mathrm{LiH}$ and $\mathrm{Li}_{2}$ molecules. There is good agreement between the numerical values of

TABLE V. Orbital energies (in hartrees) for the Be atom and the $\mathrm{LiH}$ and $\mathrm{Li}_{2}$ molecules. Numerical results for the xOEP are presented [9]. All STO (GTO) calculations have been done with the VB3 (cc-pV5Z) basis set. The threshold $\varepsilon$ used in the TSVD to discriminate the eigenvalues of the $\mathbf{M M}^{\dagger}$ matrix in the XOEP method was $10^{-5}$.

\begin{tabular}{|c|c|c|c|c|c|}
\hline & HF-STO & HF-GTO & xOEP-numeric & xOEP-STO & xOEP-GTO \\
\hline & & & $\mathrm{Be}$ & & \\
\hline $1 s$ & -4.17045 & -4.17326 & -4.1668 & -4.1689 & -4.1704 \\
\hline \multirow[t]{2}{*}{$2 s$} & -0.34903 & -0.34925 & -0.30885 & -0.3081 & -0.3082 \\
\hline & & & $\mathrm{LiH}$ & & \\
\hline $1 \sigma$ & -2.44543 & -2.44534 & -2.0786 & -2.09092 & -2.07071 \\
\hline \multirow[t]{2}{*}{$2 \sigma$} & -0.30172 & -0.30172 & -0.3011 & -0.31391 & -0.31384 \\
\hline & & & $\mathrm{Li}_{2}$ & & \\
\hline $1 \sigma_{g}$ & -2.45311 & -2.44994 & -2.0276 & -2.00774 & -2.01361 \\
\hline $1 \sigma_{u}$ & -2.45311 & -2.44994 & -2.0272 & -2.01262 & -2.00738 \\
\hline $2 \sigma_{g}$ & -0.18194 & -0.18193 & -0.1813 & -0.18401 & -0.18616 \\
\hline
\end{tabular}

TABLE VI. Decomposition of the total energy (in hartrees) in its terms for the Be atom and the CO molecule. All STO (GTO) calculations have been done with the VB3 (cc-pV5Z) basis set. The threshold $\varepsilon$ used in the TSVD to discriminate the eigenvalues of the $\mathbf{M M}^{\dagger}$ matrix in the xOEP method was $10^{-5}$.

\begin{tabular}{|c|c|c|c|c|c|}
\hline & HF-Numeric & HF-STO & xOEP-STO & HF-GTO & xOEP-GTO \\
\hline & \multicolumn{5}{|c|}{$\mathrm{Be}$} \\
\hline$T_{s}$ & & 14.57301 & 14.57319 & 14.57301 & 14.57309 \\
\hline$E_{\text {nuc }}$ & & -33.63509 & -33.63271 & -33.63518 & -33.63387 \\
\hline$E_{b i}{ }^{\mathrm{a}}$ & & 4.48911 & 4.48964 & 4.48916 & 4.48944 \\
\hline \multirow[t]{2}{*}{$E_{\mathrm{tot}}$} & $-14.57304^{b}$ & -14.57300 & -14.57254 & -14.57301 & -14.57255 \\
\hline & \multicolumn{5}{|c|}{$\mathrm{CO}$} \\
\hline$T_{s}$ & & 112.64045 & 112.63314 & 112.64200 & 112.63348 \\
\hline$E_{\text {nuc }}$ & & -310.87673 & -310.86921 & -310.88020 & -310.86944 \\
\hline$E_{b i}{ }^{\mathrm{a}}$ & & 62.93165 & 62.93421 & 62.93349 & 62.93637 \\
\hline$E_{\mathrm{tot}}$ & $-112.79078^{c}$ & -112.79056 & -112.78526 & -112.79064 & -112.78551 \\
\hline
\end{tabular}

\footnotetext{
${ }^{\mathrm{a}}$ This term is the sum of the Hartree and the exchange energies.

${ }^{\mathrm{b}}$ Reference [34].

${ }^{\mathrm{c}}$ Reference [35].
} 


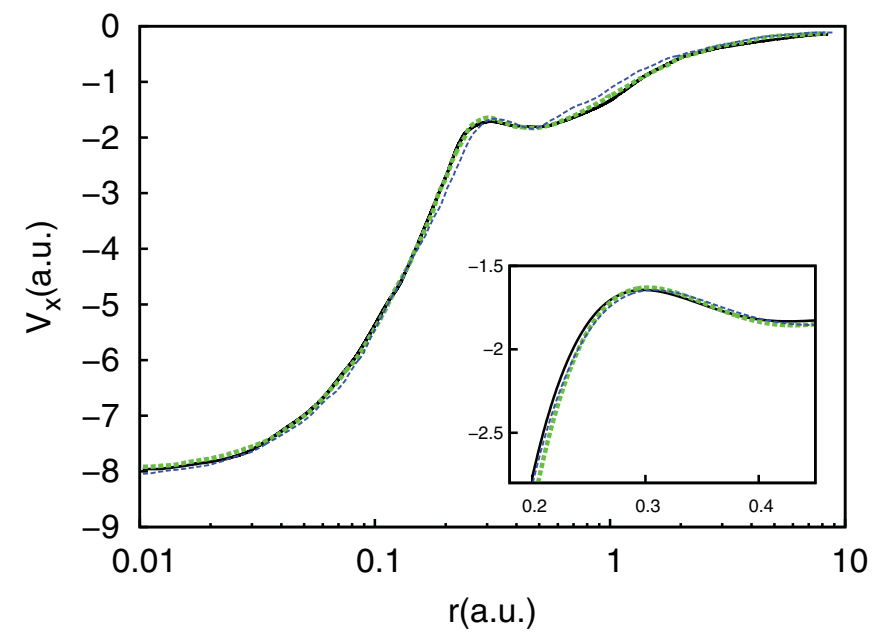

FIG. 1. (Color online) xOEP potential for the Ne atom. Atomic units are used in both coordinate axes as well as a logarithmic scale in the OX axis. The dashed (blue) line corresponds to the xOEP-GTO calculation of Fernández et al. [31]; the solid (black) line represents the results of this paper and the dotted (green) line reflects the numerical results of Kurth and Pittalis [36]. In the inset (where the OX scale is now linear) we show in more detail the region around the shoulder.

these orbital energies and the energies obtained with the two xOEP methods. Furthermore, the orbital energies from the xOEP-STO and from the xOEP-GTO calculations agree with each other to within a few $\mathrm{mHa}$.

In addition to the total and orbital xOEP energies, we have also studied the energy decomposition into the kinetic, nuclear-electron attraction, electron-electron repulsion, and the exchange energies. Table VI presents the above components of the total xOEP energy as obtained using the STO and GTO basis sets for the $\mathrm{Be}$ atom and the $\mathrm{CO}$ molecule (there are no numerical solutions available in this case for the $\mathrm{xOEP}$ ).

Figures 1 and 2 show the results of the xOEP potential for the $\mathrm{Ne}$ atom and the $\mathrm{CO}$ molecule along the main axis. For the $\mathrm{Ne}$ atom, note the close agreement between our result and that obtained with the exact numerical calculation by Kurth and Pittalis [36], thus yielding a smooth potential that shows only small deviations from the numerical potential; in any case, these xOEP potentials are also very similar to that evaluated with a Gaussian basis set [31] using the procedure presented in Refs. [20,21]. In the lower panel of Fig. 2 the results we have obtained using the VB3 basis set for the CO molecule are compared with the calculation by Heßelmann et al. [22], using an auxiliary basis set within a Gaussian representation; the STO results show a good agreement with the XOEP-GTO potential. For the sake of completeness, in the upper panel we also present several other calculations for the internuclear region, using STO basis sets of different quality (VB1, VB2, and VB3). Note that our xOEP potentials do not present any unphysical wiggle as those found by Staronerov et al. [18] and have a good agreement with both the numerical calculation and the xOEP-GTO solution by Fernández et al. [31] and by Heßelmann et al. [22] with the use of auxiliary basis set.

In summary, the previous results show that both xOEP-STO and xOEP-GTO methods yield results close to the numerically
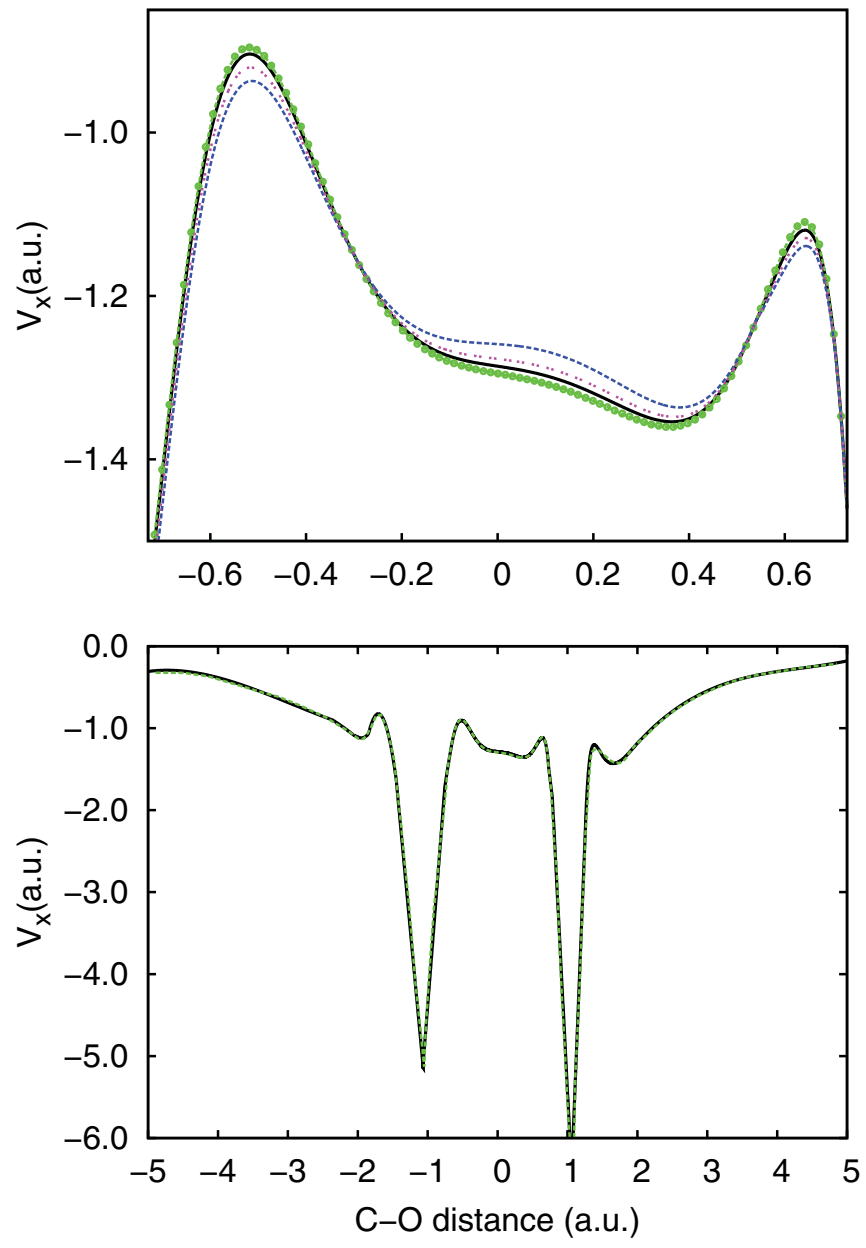

FIG. 2. (Color online) xOEP potential for the $\mathrm{CO}$ molecule along the axis of the molecule. Atomic units are used in both coordinate axes (we have used an internuclear distance of 2.132 a.u.). In the lower panel, the solid black line shows the results of this paper and the dotted (green) one reflects the XOEP-GTO results of Heßelmann et al. [22]. In the upper panel the region between the nuclei of the molecule is depicted in more detail; the xOEP-GTO results are shown again in green, and results for xOEP-STO calculations with different STO basis sets [28] are also plotted (VB1, dashed blue line; VB2, dotted magenta line; VB3, solid black line).

exact ones for the total energies, the one-electron energies of the occupied orbitals, and the XOEP potentials.

\section{CONCLUSIONS AND DISCUSSION}

The implementation of the xOEP formalism with Slatertype basis functions has been developed. This procedure has been tested in a small group of closed-shell atoms and diatomic molecules, for which both numerical xOEP solutions and STO basis sets are available. When compared to the exact numerical solution of the $\mathrm{xOEP}$ equations we have obtained very good results; they are even a bit better than those given by the Gaussian-type basis-set procedure. On the other hand, both xOEP-STO and xOEP-GTO results obtained with the prescription proposed in Refs. [20] and [21] give energies about $0.1 \mathrm{mHa}$ below those obtained for xOEP-GTO by Heßelmann et al., and thus they are closer to the exact results [9]. 
The new method leads to a considerably more compact expansion space for the xOEP local multiplicative potential, yielding noticeable savings in the computational effort to be done in each of the cycles of the self-consistent procedure. Yet another advantage of using the Slate-type basis sets is that, within the TSVD algorithm, fewer eigenvalues of the (near) singular matrix $\mathbf{M M}^{\dagger}$ need to be employed, which leads to an increased numeric stability of the XOEP-STO method as compared to the XOEP-GTO algorithm.

As a final remark, it is known that a more efficient xOEP algorithm can be developed based on the use of the incomplete Cholesky decomposition technique. The use of this technique would facilitate the application of the XOEP-STO method to larger molecules. This implementation is currently in progress and will be reported elsewhere.
On the other hand, this work is a first step to develop a local potential formalism for both exchange and correlation. Due to the smaller memory requirements of the STO scheme we have presented here, it can be used to study bigger molecules than those that can be solved with the standard GTO approach. The implementation of the correlation part of the potential is under development.

\section{ACKNOWLEDGMENTS}

The authors thank Professor Jaime Fernández Rico and his research group for providing a copy of the SMILES suite of programs and supporting this work. The authors acknowledge the financial support from the Spanish Ministerio de Ciencia e Innovación through research project Grant No. FIS201021282-C02-02.
[1] P. Hohenberg and W. Kohn, Phys. Rev. 136, B864 (1964).

[2] W. Kohn and L. J. Sham, Phys. Rev. A 140, A1133 (1965).

[3] S. Kummel and L. Kronik, Rev. Mod. Phys. 80, 3 (2008).

[4] R. T. Sharp and G. K. Horton, Phys. Rev. 90, 317 (1953).

[5] J. D. Talman and W. F. Shadwick, Phys. Rev. A 14, 36 (1976).

[6] E. Engel and R. M. Dreizler, J. Comp. Chem. 20, 31 (1999).

[7] V. Sanhi, J. Gruenebaum, and J. P. Perdew, Phys. Rev. B 26, 4371 (1982).

[8] E. Engel and S. H. Vosko, Phys. Rev. A 47, 2800 (1993).

[9] A. Makmal, S. Kümmel, and L. Kronik, J. Chem. Theory Comput. 5, 1731 (2009).

[10] M. Stadele, M. Moukara, J. A. Majewski, P. Vogl, and A. Görling, Phys. Rev. B 59, 10031 (1999).

[11] T. Kördorfer, S. Kümmel, and M. Mundt, J. Chem. Phys. 129, 014110 (2008).

[12] E. Engel and R. N. Schmid, Phys. Rev. Lett. 103, 036404 (2009).

[13] A. Makmal, R. Armiento, E. Engel, L. Kronik, and S. Kümmel, Phys. Rev. B 80, 161204(R) (2009).

[14] E. Engel, Phys. Rev. B 80, 161205(R) (2009).

[15] J. B. Krieger, Y. Li, and G. J. Iafrate, Phys. Rev. A 46, 5453 (1992).

[16] Y. Li, J. B. Krieger, and G. J. Iafrate, Phys. Rev. A 47, 165 (1993).

[17] F. Della Sala and A. Görling, J. Chem. Phys. 115, 5718 (2001).

[18] V. N. Staroverov, G. E. Scuseria, and E. R. Davidson, J. Chem. Phys. 125, 081104 (2006).

[19] S. Ivanov, S. Hirata, and R. J. Bartlett, Phys. Rev. Lett. 83, 5455 (1999).
[20] C. Kollmar and M. Filatov, J. Chem. Phys. 127, 114104 (2007).

[21] C. Kollmar and M. Filatov, J. Chem. Phys. 128, 064101 (2008).

[22] A. Heßelmann, A. W. Götz, F. Della Sala, and A. Görling, J. Chem. Phys. 127, 054102 (2007).

[23] A. Görling, Phys. Rev. Lett. 83, 5459 (1999).

[24] W. Yang and Q. Wu, Phys. Rev. Lett. 89, 143002 (2002).

[25] A. Görling, A. Heßelmann, M. Jones, and M. Levy, J. Chem. Phys. 128, 104104 (2008).

[26] R. Colle and R. K. Nesbet, J. Phys. B 34, 2475 (2001).

[27] J. E. Harriman, Phys. Rev. A 27, 632 (1983).

[28] J. Fernández Rico, I. Ema, R. López, G. Ramírez, and K. Ishida, Recent Advances in Computational Chemistry: Molecular Integrals over Slater Orbitals (Transworld Research Network, Kerala, India, 2008), Chap. 5.

[29] T. Heaton-Burgess, F. A. Bulat, and W. Yang, Phys. Rev. Lett. 98, 256401 (2007).

[30] S. Ivanov, S. Hirata, and R. J. Bartlett, J. Chem. Phys. 116, 1269 (2002).

[31] J. J. Fernández, C. Kollmar, and M. Filatov, Phys. Rev. A 82, 022508 (2010).

[32] J. E. Harriman, Phys. Rev. A 34, 29 (1986).

[33] H.-J. Werner et al., MOLPRO, version 2010.1 (2010).

[34] C. F. Bunge, J. A. Barrientos, A. V. Bunge, and J. A. Cogordan, Phys. Rev. A 46, 3691 (1992).

[35] F. Jensen, Theor. Chem. Acc. 113, 187 (2005).

[36] S. Kurth and S. Pittalis, in Computational Nanoscience: Do It Yourself!, NIC Series, Vol. 31 (John von Neumann Institute for Computing, Julich, 2006), pp. 299-334. 